\title{
Electromagnetic Coupling of Ocean Flow with the Earth System
}

\author{
Robert Tyler ${ }^{1,2, *}$ \\ ${ }^{1}$ Department of Astronomy, University of Maryland, College Park, USA \\ ${ }^{2}$ Planetary Geodynamics Laboratory, NASA's Goddard Space Flight Center, Greenbelt, USA
}

Received 23 May 2013, revised 21 January 2014, accepted 19 August 2014

\begin{abstract}
The ocean is electromagnetically coupled with the Earth System. This results in momentum transfer, as well as a participation by the ocean in the Earth's observable electric and magnetic fields. The coupling is typically quite weak and quantitative analyses indicate that many of these connections may be discounted when considering the transfer of momentum. But because of systematic effects there are also cases where an immediate discount is not justified and electromagnetic transfer of ocean momentum should remain within the realm of consideration. For practical considerations, even if the coupling is weak these effects are phenomenologically important because the electric and magnetic fields associated with this coupling offer an observational means for inferring the ocean flow. While in situ measurements of the electric field have long been used to measure ocean transport, new opportunities for remote sensing ocean flow through ground and space magnetic observatories are now being considered. In this article a brief update of the status of these observational methods is given. Extending beyond these established elements of the ocean's electromagnetic involvement, an attempt is made to provide a quantitative discussion of lesser considered elements of the ocean's electromagnetic coupling with the mantle and fluid core.
\end{abstract}

Key words: Electrodynamics, Induction, Ocean flow, Electromagnetic coupling

Citation: Tyler, R., 2015: Electromagnetic coupling of ocean flow with the Earth system. Terr. Atmos. Ocean. Sci., 26, 41-52, doi: 10.3319/ TAO.2014.08.19.04(GRT)

\section{INTRODUCTION}

As early as the experiments of Michael Faraday it could be inferred that the Earth comprises an electromagnetic system, a system that interacts with the Earth's system of material momentum. Phenomenologically, the Earth's electromagnetic and material momentum systems are coupled; there is continuous energy exchange between material kinetic energy and the electric currents/electromagnetic fields permeating the Earth System. The angular momentum of the core, mantle, ocean, and atmosphere, for example, are electromagnetically coupled, with the expected tendency for relative rotation between these elements to be reduced by these electromagnetic forces. But the efficiency of the coupling (i.e., the time scale for momentum transfer) depends on material parameters such as electrical conductivity that vary by about eighteen orders of magnitude within the Earth System, likely leaving aspects of this coupling to be phenomenologically unimportant and unobserved.

\footnotetext{
* Corresponding author

E-mail:robert.h.tyler@nasa.gov
}

Formulatively, the coupling is apparent when the "coupling" terms are retained in the governing equations for the momentum dynamics and the electrodynamics and the two systems of equations must be solved simultaneously. But there are also cases where the "coupling" is, somewhat oxymoronically, one directional. Outside of the core and below the upper atmosphere, it is typically the case that the electromagnetic energy density is much smaller than the energy density associated with the momentum in the material. This disparity is an important consideration when considering the electrodynamics and electromagnetic coupling in the ocean. A very small fraction of the flow kinetic energy converts into an appreciable fraction of the electromagnetic energy density. From this, one may expect that the electromagnetic forces on the material are negligible over short time scales as they would have to act for a very long time to transfer enough momentum to the material to alter its momentum balance.

The participation of ocean flow in the Earth's electromagnetic system then appears as follows: the ocean flow interacts with the Earth's main magnetic field to generate 
secondary electric currents and electric and magnetic fields; and through electromagnetic coupling, the ocean may pass momentum to other elements of the Earth System with subsequent additional electromagnetic effects (see Fig. 1). The first element is of interest because it offers a new potential for monitoring ocean flow through remote observations of the ocean flow generated magnetic fields. Progress on this element shall be reviewed in the next section. The second element, the passage of momentum to other components of the Earth System through electromagnetic coupling, has received little attention because simple estimates show the coupling should be quite weak. But there are nonetheless interesting considerations that can be made when considering long timescales, and these shall be discussed in section 3 .

\section{MAGNETIC REMOTE SENSING OF OCEAN FLOW}

As described in the Introduction, the ocean is quite unlike the fluid core or upper atmosphere in that the energy density of the electromagnetic field is quite small in comparison to the energy density contained in kinetic and other forms. From this energy density disparity, one may note that because momentum fluctuations are exaggerated in their manifestation in the electromagnetic field, and because the electromagnetic field can be measured within the ocean or remotely, there are immediately favorable characteristics in monitoring ocean flow through observations of the flow induced electromagnetic fields. Ocean flow is, since long, inferred from measurements of the electric field, either in the ocean (e.g., Larsen and Sanford 1985) or on land (e.g., Junge 1988). Ocean flow also generates magnetic fields which have been measured on land (e.g., Larsen 1968; McKnight 1995; Maus and Kuvshinov 2004; Manoj et al. 2011) in the ocean (e.g., Larsen and Cox 1966; Lilley et al. 1986), in aeromagnetic surveys (e.g., Weaver 1965), and by satellite magnetometers (Tyler et al. 2003). Work to infer ocean flow from aero- and satellite-magnetic surveys is highly motivated for three reasons: (1) in situ methods for monitoring the flow typically under sample the horizontal dynamical spatial scales, and conventional remote-sensing methods lack resolution of vertically integrated flow parameters; (2) satellite data describing the Earth's magnetic field in unprecedented resolution is recently available, it is expected to continue and improve, and this data contains detectable signals describing large-scale ocean flow; (3) aeromagnetic surveys may feasibly retrieve information about meso-scale ocean variability which is currently a limiting factor in all ocean/climate models.

Within the last decade the Earth's magnetic field has been observed in unprecedented spatial resolution by spaceborne magnetometers (i.e., the low-Earth orbiting Oersted launched in 1999; CHAM and SAC-C launched in 2000) and this trend toward high-precision surveys of the geo- magnetic field from space are expected to continue with the launch of Swarm (Haagmans 2005) by the European Space Agency in November 2013. In fact, an objective in Swarm is to surpass the unprecedented resolution gained from these recent space borne surveys and deliver magnetic signals descriptive of ocean flow and climate changes. Swarm will be the most sophisticated magnetic observatory to date as it shall consist of three coordinated satellites - two orbiting at $450 \mathrm{~km}$ altitude, and one at $550 \mathrm{~km}$ - which shall provide both direct and differential observations of the Earth's vector magnetic field. The improved observational base of the Earth's magnetic field, through allowing improved models of the geomagnetic field, improves the signal-to-noise ratio associated with remaining ocean flow magnetic signals. The nature and sources of the other signals confounding extraction of the ones due to ocean flow depend on the temporal periods considered. At periods of a few days and shorter, magnetic fields due to electric currents in the ionosphere and magnetosphere are typically larger than those generated by ocean flow. These external components might be separated from the internal ones (e.g., those generated by ocean flow) in a spherical-harmonic expansion, or by fitting the external sources to prescribed base functions involving a reduced number of parameters (e.g., Olsen et al. 2010). One may note that these external magnetic fields excite, however, electric currents (and associated magnetic fields) in the ocean and near-surface earth. The external magnetic fields

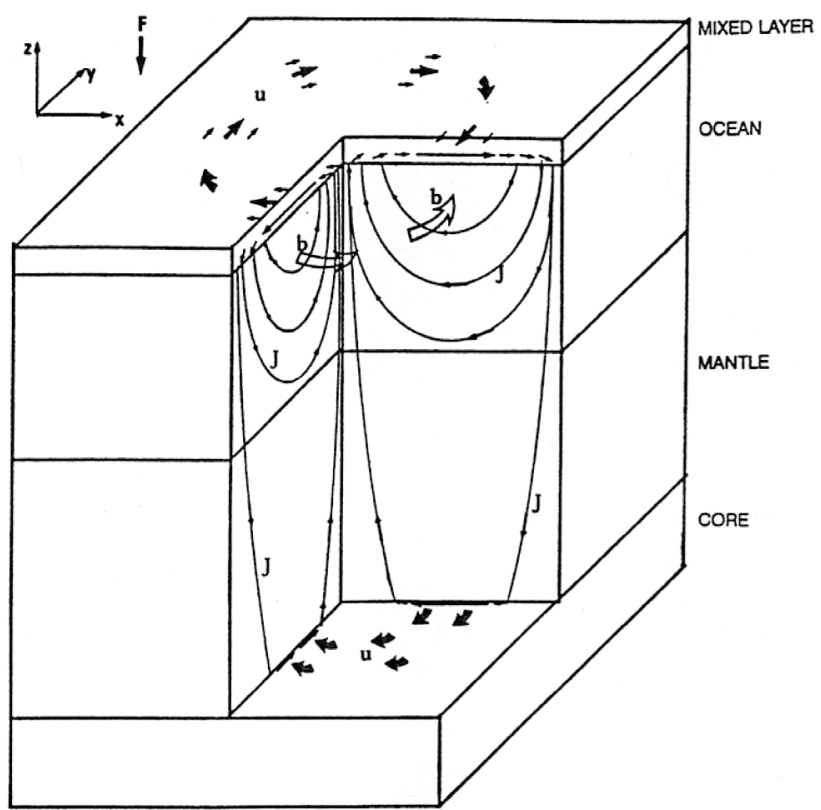

Fig. 1. A simple example of an electromagnetic coupling involving the ocean: a surface intensified ocean gyre involves strong flow $\mathbf{u}$ in the surface mixed layer which interacts (through the Lorentz term $\mathbf{u} \times \mathbf{F}$ ) with the Earth's main magnetic field $\mathbf{F}$ to generate electric currents $\mathbf{J}$ and associated magnetic fields b. Electric currents close through the lower ocean, mantle, and core, coupling these layers and transferring momentum from the surface flow to the lower layers. 
thereby excite their own internal components, leading to an additional challenge in separating the oceanic signals. The external signals and their excited internal component are of course related in a predictable way (e.g., Kuvshinov 2008) and it is clear that prospects for extracting the ocean-flow signals improve with better observational coverage and improved geomagnetic field modeling.

A second development aiding the identification of the ocean-flow signals in the magnetic record is the use of theory and numerical simulation to determine predictable aspects of the expected oceanic signals that can be used statistically in identifying (separating/extracting) these signals in the magnetic record. Much of this is based in earlier work aimed primarily at exploiting the in situ electric field and involves calculations for idealized cases (important early references include Longuet-Higgins et al. 1954; Cox et al. 1971; Sanford 1971; Larsen 1973; Podney 1975; Chave 1983). Toward more realistic descriptions with focus on the magnetic field, Stephenson and Bryan (1992) performed the first numerical calculations of the electric and magnetic fields generated by global ocean model circulation. This was immediately followed by a series of studies that have reiterated the earlier findings and included theoretical and numerical extensions. Tyler and Mysak (1995), Tyler et al. (1997a, b) included theoretical and numerical descriptions of the stronger toroidal component of the magnetic field within the three-dimensional (3-D) global ocean, and Tyler et al. (1999) described the expected satellite-altitude magnetic signal associated with global ocean circulation. By now, simulations using a diversity of ocean model circulation and at least two substantially different numerical schemes for calculating the flow generated magnetic fields have shown basic agreement on the amplitudes and patterns of the ocean circulation generated magnetic fields at the sea surface and satellite altitudes, and have improved understanding of the effects of model resolution in these predictions as well as the expected temporal variability [see Glazman and Golubev (2005), Manoj et al. (2006), Kuvshinov (2008) for recent reviews, and further references included below]. The amplitude of the magnetic fields due to the steady component of circulation, while $10-100 \mathrm{nT}$ within the ocean, is typically $1-10 \mathrm{nT}$ at the sea surface and a few $\mathrm{nT}$ at an altitude of $500 \mathrm{~km}$. These small amplitudes fall within that of lithospheric and crustal magnetic anomalies and are presently difficult to distinguish.

Even the temporally varying components, while surely distinct from lithospheric/crustal components, have remained elusive because of the small signal-to-noise ratio of the expected oceanic magnetic signals and because more work is needed to determine the signatures of these oceanic magnetic signals so that an extraction is possible. In the case where the signature is very predictable (e.g., tides) a successful extraction has already been performed; In Tyler et al. (2003), the magnetic field due to the predominant $\mathrm{M}_{2}$ tide were numerically calculated and correctly predicted the global distribution in phase and amplitude of the $\mathrm{M}_{2}$ signal which was then derived independently from satellite magnetic observations. In unpublished work, the author has found that with even a simplistic inversion scheme (based on flow divergence), the primary pattern of the global tides can be recovered from the synthetic magnetic data (i.e., the magnetic fields calculated from the model tides). Kuvshinov et al. (Vennerstrom et al. 2005) using also a simplistic inversion scheme (based on flow rotation) demonstrate feasibility in inverting synthetic magnetic signals to recover large-scale ocean circulation. It has also been shown in the three different simulations in Vivier et al. (2004) that an important variable in ocean/climate research, the fluctuating strength of the Antarctic Circumpolar Current (ACC) transport, can be easily inferred from space-borne magnetometers-provided the relatively weak ACC signal can be extracted from competing signals in the record. Tsunami sea-surface height and transport can in principle be inferred from aero-, satellite-, and seafloor-magnetic observations (Tyler 2005). While there has indeed been some recent success in this (Hamano et al. 2011; Manoj et al. 2011; Toh et al. 2011; Utada et al. 2011; V. Klausner, pers. comm.), the inference involves only basic amplitude consistency comparisons that do not yet demonstrate feasibility. In addition to the magnetic fields generated by the tsunami flow, the tsunami sea surface displacement also excites acoustic and gravity waves which propagate upward through the atmosphere and into the ionosphere (Kherani et al. 2006; Occhipinti and Kherani 2008; Occhipinti et al. 2013). While the coupling through the lower atmosphere is not electromagnetic (the focus of this paper), the result is however related in that there is an excitation of electric currents in the ionosphere with associated magnetic fields caused by the ocean tsunami.

These various ocean flow signals are detectable by magnetometers but are not typically easy to identify in the record. The basic challenge is that the ocean flow magnetic fields show significant spatio-temporal overlap with the fields of other unresolved processes of similar or greater amplitude. Indeed, the successes in identifying ocean flow magnetic signals have so far occurred only in the extreme ends of either stationary (tides) or non-stationary (tsunami) processes where this overlap is minimized.

Several complimentary approaches may be used to address this issue: first, there is rapid improvement in the accuracy of models of the Earth's magnetic field and the oceanic magnetic signals must only be distinguished from the unresolved "noise", rather than the primary signals. Second, ocean flow and the generated magnetic field are constrained both by dynamics and by a variety of observations, and these constraints can be used to develop statistical tools for extracting the oceanic signals. In the case of the tides, the known frequency provided an obvious extraction tool; a repeating signal can be averaged over its cycles to improve the signal-to-noise ratio. In the case of lower-frequency 
ocean flow variations, an extraction tool is not as immediate. Much is known about these lower-frequency variations, this includes dynamical constraints and observations, and the realistic descriptions given by data-assimilating ocean models which combine dynamical and observational constraints. Finally, bases functions for the ocean flow sources may be developed and used to co-estimate the oceanic contributions in magnetic field models that simultaneously fit magnetic data to multiple sources.

\section{THE BROADER ROLE OF THE OCEAN IN THE EARTH'S ELECTROMAGNETIC SYSTEM}

The elements of the ocean's role in the Earth's magnetic field described in the last section are firmly established and work is aimed not at the fundamental processes but rather at methodologies for connecting and exploiting these elements. There are in principle, however, other roles in which the ocean may participate electromagnetically with other components of the Earth which shall now be discussed. It is far from certain that any of these exotic elements are significant but it is also not clear that they can be dismissed in all applications. At the very least, an attempt to quantify the coupling involved is helpful.

In the Introduction we described that because only a small proportion of the oceanic energy is carried in the electric currents and electromagnetic field, the electromagnetic effects on material momentum can be ignored. In research by the author (Tyler 1995; Tyler and Sanford 1998; Tyler 2006), a recent study (Ryskin 2009), and results presented here, this assumption is challenged. In Tyler (2006) it was shown that while the electromagnetic Lorentz forces on the global ocean circulation are indeed weak, they are not immediately negligible as they impose systematic forces over long time scales. If other forces were to stop, the time scale for these electromagnetic forces to brake ocean flow (primarily large-scale heat transport) is about 3000 years (Tyler 2006). Interestingly, this time scale decreases inversely with the square of the main magnetic field amplitude, such that the Earth's magnetic field could not be more than about an order of magnitude larger than its current strength before it would likely have indisputable effects on ocean transport and climate. It is also known that the magnetic field shields life from high-energy particles from space, and that the strength of the field in the geological past has typically been similar to that at present (Hulot et al. 2010). Combining these elements, an interesting result arrives: it seems that the Earth's magnetic field has typically maintained a strength that is large enough to provide shielding but not too large as to appreciably brake oceanic heat transport and drastically affect climate. This 3000-year time scale also describes the time scale for transfer of ocean angular momentum to the mantle through electromagnetic coupling.

In another example of broader connections, Tyler
(1995) pointed out that the pattern of homogenization of the radial component of the main magnetic field [as down-cast to the core-mantle boundary (CMB)] in the regions of the North Pacific and North Atlantic were consistent with diffusive entrainment of the surface core fluid by electromagnetic coupling with known circulation gyres in the oceans above. It was also pointed out that the coupling was, however, extremely weak. In Tyler and Sanford (1998), it was estimated that systematic poleward transport in the oceans leads to weak but systematic Lorentz forces in the core. In a recent paper that received wide attention (Ryskin 2009) it was proposed that ocean flow is responsible for the secular variation (SV) in the Earth's magnetic field. [The author has carefully reviewed Ryskin (2009) and while some of the supporting correlations are interesting it seems that there are several theoretical flaws in the proposed mechanism, and a more immediate problem is that it would require electric currents in the ocean that are much stronger than what has been observed.]

In the following subsections, we shall compile original elements of previous work by the author to provide quantitative estimates for the weak coupling of the ocean circulation with the mantle and fluid core. In this case, the oceans may affect the geomagnetic field over only very long time scales. The calculated oceanic forces on the core are so weak that if not for corroborating observational evidence, it would seem justifiable to assume that the effect of these forces, while systematic over long time scales, are still lost in the effects of more pervasive forces. But, as will be shown below, the pattern of core flow estimated from these oceanic forces shows correspondence with flow features derived independently from inversion of magnetic data.

There are a variety of ocean circulation models that can be used for such study and the choice will depend on the aspect of the study being addressed. Several different models have been used in the global simulations (as cited above). Regional models typically provide higher resolution and more physical realism as less of the dynamics are parameterized, and one expects then that these can be used to more robustly determine the physical relationships between the magnetic fields and other variables. But even in this case there are choices that must be carefully considered because the approach used in the dynamics and data melding (in assimilation models) are aimed at differing optimizations in satisfying dynamical and observational constraints. While some models gain improved descriptive performance by nudging toward observations or climatologies (at the sacrifice of full dynamical consistency), the goals in the magnetic applications are probably better met by models that retain consistent dynamics. An appropriate choice for the ACC simulations, for example, is the recent ECCO-GODAE ocean description (Mazlof et al. 2010) which assimilates a comprehensive set of observations but formulatively retains full dynamical consistency. Through subsequent 
comparison studies with other ocean models it can be determined whether model dynamical consistency is crucial in preserving the correct physical connection with the oceanic magnetic fields. In the examples below, the ocean circulation used is the time-mean of the ECCO ocean circulation used by the author in Vivier et al. (2004).

\subsection{Ocean/Mantle Coupling}

It has long been known that the Earth's angular velocity varies both in amplitude (e.g., rotation rate) and in orientation (e.g., polar motion). These variations are the manifestation of interactions among different systems of the Earth, and among the Sun-Earth-Moon system. For example, the Earth's rotation rate, perhaps represented by the length of day (LOD), varies over a wide time spectrum, with fluctuations varying over a few days to geological time scales. Past studies have indicated that the couplings between the solid Earth and the surface geophysical fluid systems (e.g., atmosphere and ocean) account for the Earth's angular velocity variation on daily to inter-annual time scales (Salstein et al. 1993; Marcus et al. 1998; Gross et al. 2003, 2004, 2005; Dehant et al. 2005). Over decadal time scales and perhaps longer, the coupling between the solid mantle and the fluid outer core is thought to be the dominant mechanism for the Earth's rotation variation (e.g., Jault and Le Mouël 1993; Holme and Whaler 2001). However, the longer period (e.g., millennium time scales) SV in the Earth's rotation is attributed to SunEarth-Moon tidal breaking, and post-glacial rebound which changes the moment of inertia of the solid Earth (e.g., Stephenson and Morrison 1995). In fact, longer period SVs in the Earth's rotation have been used to constrain the mantle viscosity (e.g., Peltier and Drummond 2010).

For the short time scales of days to decades, studies of the contribution of oceanic dynamical processes on the Earth's rotation variation have considered the topographic coupling due to dynamical pressure at the ocean bottom, and the changes in the moment of inertia associated with ocean circulation and tides (Gross et al. 2004, 2005; Gross 2009). Electromagnetic coupling is much weaker but could be im- portant on longer timescales, or in coupling the ocean with deeper regions; one expects a finite electromagnetic torque on the solid mantle by the ocean: $\Lambda=\iiint_{V} \hat{\mathbf{r}} \times(\mathbf{J} \times \mathbf{B}) d V$ where $\mathrm{V}$ is the volume of the solid Earth, $\mathbf{B}$ and $\mathbf{J}$ are the magnetic field and current density, respectively. In terms of their fractions of back ground values, the ocean-generated electric currents are larger than the ocean-generated magnetic fields, and so in the integral let us presume that $\mathbf{J}$ is specifically driven by ocean flow, while $\mathbf{B}$ may be taken to be the background main field. This torque will excite rotational changes in the solid Earth, or at least systematic forces over long time scales exceeding that of geomagnetic reversals (because of invariance of the Lorentz forces to sign reversal in the magnetic field) and feasibly reaching even the geological time scales (10 - $100 \mathrm{Myr})$ describing the age of ocean basins. Certainly the dominant patterns of ocean circulation (including the mid-latitude gyres, and the ACC) reflect the wind stress curl effected by basic atmospheric convective cells acting since very long.

Estimates of this torque depend very much on the electrical conductivity assumed for the mantle. But a simple result can be provided here that the readers may use together with their own conductivity assumptions to become convinced of the very small amplitude of the torque. In Fig. 2, we show a surface map of the electric potential generated by the ocean circulation and 3-D electromagnetic model discussed in the next section. The basic amplitude and distribution of the electric potential is not overly sensitive to the conductivity assumed under the ocean so long as the resistance remains high. It is this distribution of electric potential that drives electric currents to close through paths beneath. The amplitude of the voltage difference is only of order $1 \mathrm{~V}$ and the associated electric field in the mantle is of order $10^{-6} \mathrm{~V} \mathrm{~m}^{-1}$. If one assumes a bulk mantle/lithosphere electrical conductivity value of $10^{-3} \mathrm{~S} \mathrm{~m}^{-1}$, say, the electric current density is then of order $10^{-9} \mathrm{~A} \mathrm{~m}^{-2}$.

\subsection{Ocean/Core Coupling}

It can be expected that angular momentum transferred

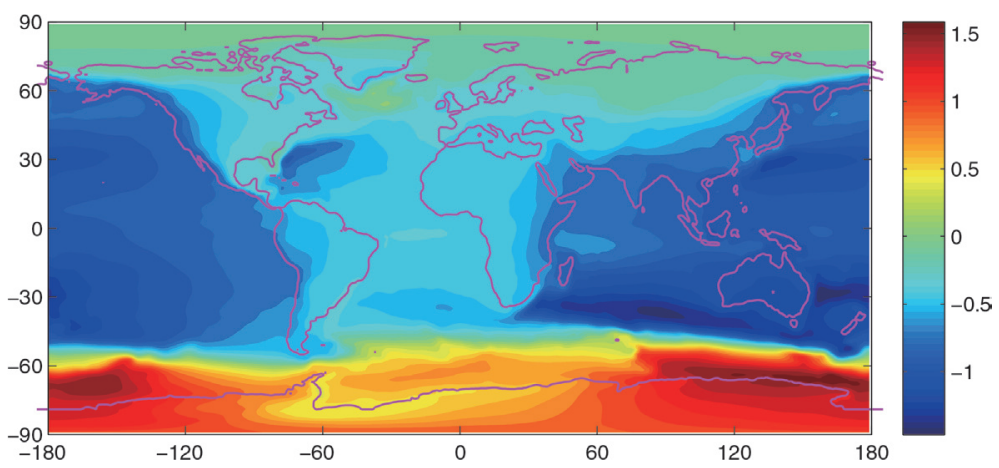

Fig. 2. Electric potential (V) generated using time mean of ECCO model ocean circulation. 
from the ocean to the mantle can be further transferred to the fluid core through further core/mantle electromagnetic coupling. But there is also no doubt that a portion of the ocean-flow generated electric currents pass directly through the electrically conducting solid Earth and into the Earth's fluid core, and that the cross product of this electric current density, $\mathbf{J}$, and the ambient magnetic field, $\mathbf{B}$, provides an electromagnetic (Lorentz) force $\mathbf{J} \times \mathbf{B}$ causing accelerations in the core fluid. It is clear, however, from even a simple scaling analysis that these oceanic forces on the core should be extremely weak, which provides a partial explanation for why such electromagnetic coupling directly between the ocean and core has received little attention. Considered more carefully, however, it is possible that even these weak oceanic forces, when acting systematically over long timescales, can lead to significant effects and possibly observable consequences. Or at least neglect of this effect requires a justification involving the comparison of inherently secondorder terms and has not yet been performed. In either analyses, a quantitative description of these oceanic forces on the core is required and possible. Below we present an example of this estimate in three steps:

Step 1. Calculate ocean generated electric currents in core: Here we assume that the ocean is represented by the mean state in the ECCO ocean circulation model [the time mean of the simulations in Vivier et al. (2004)], and that the Earth's magnetic field is represented as an axial dipole (with amplitude $-6 \times 10^{-5} \mathrm{~T}$ at the surface geographic North pole). The assumption of an axial dipole used here may be regarded as one component of the present magnetic field or, more importantly, as the average form of the field over the long time scales between geomagnetic reversals (Hulot et al. 2010). The stability of the geographic features in global ocean circulation over long time scales is less certain though initially reasonable for time scales less than those $(10-100 \mathrm{Myr})$ describing configurational changes in the ocean basins. The electric currents in 3-D spherical geometry are calculated using a version of the Model for Ocean Electro Dynamics [MOED; Tyler et al. (2004)] configured for the quasi-static case. The domain resolution includes 70 radial layers reaching from the surface to core center, and 2-degree horizontal resolution. The 3-D electrical conductivity of the Earth is represented by an inhomogeneous surface layer comprising the layer conductance due to the ocean fluid (calculated from time averages of temperature and salinity in ECCO), and from sediment conductance calculated from sediment thicknesses and porosity estimates (as in Vivier et al. 2004). Below the surface, the radial conductivity profile of Kuvshinov is assumed and continued below $900 \mathrm{~km}$ depth to include a lower mantle of $1 \mathrm{~S} \mathrm{~m}^{-1}$, fluid core of $10^{6} \mathrm{~S} \mathrm{~m}^{-1}$, and solid inner core of $10^{6} \mathrm{~S} \mathrm{~m}^{-1}$. The radial component of the ocean generated electric current density at the CMB is shown in Fig. 3 (top left panel).
The amplitude is of the order of one $\mathrm{nA} \mathrm{m}^{-2}$, which is about three orders of magnitude weaker than it is in the ocean. This confirms, first, that very little of the ocean generated electric currents reach the core. We also see that the part that does reach the core is primarily reflective of the large-scale ACC, and the mid-latitude gyres in the North Atlantic and North Pacific.

Step 2. Calculate Lorentz forces on core surface fluid: The axial dipole magnetic field at the $\mathrm{CMB}$ has a polar amplitude of $3.8 \times 10^{-4} \mathrm{~T}$. The amplitude of the Lorentz forces $\mathbf{J} \times \mathbf{B}$ on the core fluid surface are then of the order $\left(1 \times 10^{-9} \mathrm{~A} \mathrm{~m}^{-2}\right) \times\left(4 \times 10^{-4} \mathrm{~T}\right)=4 \times 10^{-13}\left(\mathrm{~kg} \mathrm{~m}^{-3}\right)\left(\mathrm{m} \mathrm{s}^{-2}\right)$ which, dividing by core fluid density $\sim 5 \times 10^{3} \mathrm{~kg} \mathrm{~m}^{-3}$, shows the direct acceleration of the flow to be a meager $\sim 10^{-16} \mathrm{~m} \mathrm{~s}^{-2}$. This can be compared with the accelerations of order $10^{-12} \mathrm{~m} \mathrm{~s}^{-2}$ recently inferred from time variations in magnetic field observations (Olsen and Mandea 2008). (One may note that these ocean generated accelerations can be greatly amplified in time dependent cases where the electric currents have not completely diffused through the core but are instead confined to a thin surface skin-depth layer.)

Step 3. Calculate core surface flow response to these oceanic Lorentz forces: It seems that there may be a stably stratified layer near the surface of the fluid core (Gubbins 2007; Helffrich and Kaneshima 2010) which would be dynamically important in such forcing, but it is not clear what dynamical assumptions should be made in estimating the core flow produced by these oceanic Lorentz forces. In the initial approach here we shall make some simplistic dynamical assumptions which are surely incomplete but provide first estimates which include only one free parameter. We assume that the core surface flow is primarily in geostrophic balance with a pressure field $m^{*}$ supported by the long-term convergences of the Ekman transport velocity $\mathbf{u}_{E}=[\mathbf{J} \times \mathbf{B}] \times \hat{\mathbf{r}} /(\rho f)$ associated with the oceanic electric currents $\mathbf{J}$ (where $\rho$ is the core density and $f$ is the Coriolis parameter). More specifically, we assume that $m^{*}$ is a function of $\nabla \cdot \mathbf{u}_{E}$. The functional relationship we choose for this example can be represented by

$r_{\mathrm{CMB}}^{2} \nabla \cdot\left(\frac{f_{o}^{2}}{f^{2}} \nabla m^{*}\right)=-\frac{1}{f_{o}} \nabla \cdot \mathbf{u}_{E}$

where $r_{\mathrm{CMB}}$ is the radius to the $\mathrm{CMB}, f$ is the Coriolis parameter, and $f_{o}=2 \Omega$ is twice the rotation rate $\Omega$. The pressure function $m^{*}=\frac{\gamma}{\varepsilon} \frac{\widetilde{m}}{m_{o}}$ is a non-dimensionalized version of a pressure function $\bar{m}$ having units of mass per square meter. The mass density $m_{o}$ represents the unperturbed state of an assumed uniform/constant, thin dynamic layer below the $\mathrm{CMB}$; this would be obtained by integrating fluid density radially through the thin layer. In this case, $\widetilde{m}$ is viewed as 
a dynamical mass anomaly in the layer and specific forms for $\widetilde{m}$ appropriate for a variety of linear and nonlinear dynamical layer models is described in Tyler and Käse (2000a, $\mathrm{b}, 2001)$. The Lamb parameter $\varepsilon=\left(f_{o} a\right)^{2} / c^{2}$ is the squared ratio of the rotational velocity and the shallow-water wave speed $c$ associated in this case with the first baroclinic mode. The parameter $\gamma$ represents an inverse time scale (non-dimensionalized by $f_{o}$ ) for the dynamic adjustment of the primarily geostrophic state driven by $\nabla \cdot \mathbf{u}_{E}$. This time scale can be taken to represent either a Rayleigh dissipative timescale, or a time scale over which the oceanic forces in the core have been integrated. The balance in Eq. (1) is essentially an equation for the conservation of mass; in this approximation, convergence of mass by the Ekman flow is balanced by a divergence of momentum associated with small accelerations or decelerations in the geostrophic momentum. An important deficiency is that there should also be a term reflecting the divergence of momentum by long Rossby waves. This is expected to be important at least very near the Equator, but including this term would introduce at least one other free parameter to describe the unknown stratification. One can view Eq. (1) as a limiting case (appropriate for a weakly dissipative/forced, primarily geostrophic balance) of more general evolution equations for the dynamic mass anomaly $\widetilde{m}$, derivations for which are reviewed in Tyler and Käse (2001).

The geostrophic flow velocity is

$\mathbf{u}_{g}=-\frac{\left(f_{o} r_{\mathrm{CMB}}\right)^{2}}{\gamma f} \nabla m^{*} \times \hat{\mathbf{r}}$

In the form $m^{*}$ the pressure field can be calculated by direct inversion of the discretized partial differential Eq. (1).
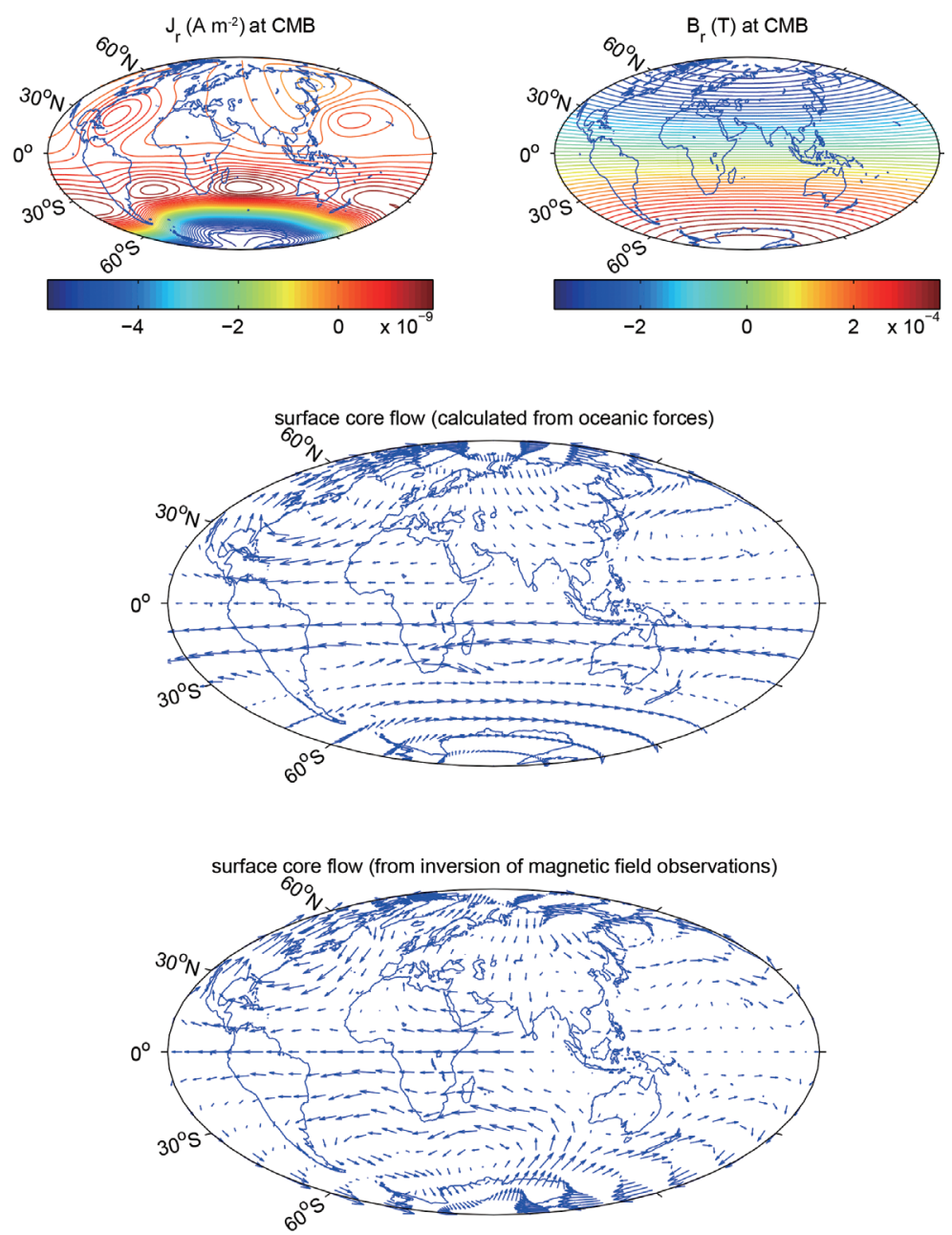

Fig. 3. Interaction of ocean with the long-term average (i.e., axial dipole) Earth's magnetic field. Radial component of ocean driven electric current density at CMB (top left panel), and the assumed main magnetic field at core-mantle boundary (CMB) (top right). The Lorentz forces associated with these electric currents and main field lead, according to a simplistic dynamical model described in the text, to the flow shown in the middle panel. Note that outside of the region of the Antarctic Circumpolar Current (ACC), there is remarkable agreement of the pattern of these flow vectors with those produced independently from magnetic field observations (bottom panel). Note, however, that any agreement of the amplitudes $\left(\sim 1 \mathrm{~mm} \mathrm{~s}{ }^{-1}\right.$, in bottom panel) is indirect because of an arbitrary time scale used in producing the flow in this study (middle panel). 
Hence, the only free (poorly constrained) parameter needed in calculating the geostrophic flow $\mathbf{u}_{g}$ using Eq. (2) is the time scale $1 / \gamma$.

The pattern of flow vectors calculated in this way are shown in Fig. 3 (middle panel) and can be compared with flow vectors derived independently by A. Jackson (pers. comm.; also see Bloxham and Jackson 1991; Jackson 1997; Holme 2007; Whaler and Holme 2007; Olsen and Mandea 2008) through inversion of observed SV in the Earth's magnetic field (bottom panel). The primary features seen in the core flow we calculate are: an equatorial westward drift due to electric currents driven by the ACC, and mid-latitude gyres corresponding to similar flow features in the ocean. At equatorial and northern latitudes, there is an interesting correspondence between the two independently derived flow patterns. Because the magnetic field assumed is quite simple, there is no chance that the match in these flow patterns is simply reiterating patterns in the main field; the structure seen is primarily due to the geometry of global ocean circulation.

In the region of the ACC $\left(<30^{\circ} \mathrm{S}\right)$ velocities have been divided by 10 for global display purposes. The flow in this region is strong and does not agree with that shown in the middle panel. The strongest oceanic Lorentz forces on the core are clearly in the region below the ACC. A potential explanation is that because the forces are much stronger in this region, the flow has accelerated much more recently and the assumption of the long-term dipole main magnetic field in calculating the Lorentz forces is inappropriate. When, instead of an axial dipole, we assume a degree- 7 geomagnetic field (describing the large features of the present field) the core flow in the region of the ACC shifts from the zonal pattern seen in the dipole case, to a primary feature involving flow across the pole and into the Indian Ocean, in agreement with the flow seen in Fig. 3 bottom panel. The amplitudes of the flow calculated from the oceanic forces agrees with the amplitude of about $10 \mathrm{~km} \mathrm{yr}^{-1}$ of flow inferred from inversions such as Fig. 3 (middle panel), provided the time scale $1 / \gamma$ is taken to be $1-10 \mathrm{Myr}$. As we have noted above, our estimates of ocean driven core flow will be an underestimate because under non-steady conditions the oceanic electric currents will be concentrated near the surface rather than spread through the core. But the indication from this initial work is that the time scale for establishing an ocean driven geostrophic flow having the advective time scales of SV is of the same order as the time scale describing reversals in the Earth's main magnetic field. Any coupling is extremely weak, but because the effect is systematic over longtime, in order to dismiss this process one would need to compare it with another process acting over the same period.

\section{DISCUSSION AND SUMMARY}

Presently, only two roles are recognized by which the ocean participates significantly in the Earth's magnetic field. First, accurate models of the inductive magnetic response of the near-surface Earth to external (ionospheric and magnetospheric source) fields has demonstrated the importance of the realistic 3-D electrical conductivity distribution in the ocean and wet sediment layers (Everett and Schultz 1996; Everett et al. 1999; Kuvshinov 2008; Kelbert et al.2009). While the importance of realistic ocean/surface conductivity for relatively high (diurnal) frequency induction modeling has long been appreciated, recent work shows that realistic modeling of the near-surface 3-D conductivity is important for induction with periods as large as 20 days (Kuvshinov 2008; Kuvshinov and Semenov 2012; Semenov and Kuvshinov 2012). This inductive effect of the ocean is simply due to its relatively high electrical conductivity and the relative motion (ocean flow) of this conductor is not involved. Another way to describe this is that the sources for the energy driving these inductive phenomena are not in the ocean nor drawn from the flow kinetic energy.

The second role of the ocean involves the generation of magnetic fields by ocean flow, and is of particular interest because of the potential for using these fields to remotely observe flow fluctuations. As described above, the small signal-to-noise ratios of these ocean flow signals presents a challenge that may be simultaneously addressed through improved modeling of competing signals, and by using predictive information about the expected oceanic signals as an extraction tool. Work is needed to develop these extraction tools for isolating the oceanic signals describing large- and meso-scale flow variations important to ocean/climate studies. This has immediate strong overlap with the basic science objective of developing a better theoretical understanding of how flow-generated magnetic fields are created and determining which aspects of the flow are most effectively represented in remote magnetic observations. From a practical consideration, there is a need not only to determine which aspects of the oceanic signals are most easily retrievable (e.g., tides) but also to focus effort on retrieving signals that would be the most useful in constraining ocean/climate models.

Some opportunities, such as the magnetic remote sensing of tsunami flow, are highly motivated but not of obvious feasibility. The expected signals [ranging from $1-20 \mathrm{nT}$ (Tyler 2005)] are indeed above the detectability level of air-and space-borne magnetometers and there are examples of detection (Manoj et al. 2011; V. Klausner, pers. comm.) which have been shown to be roughly consistent with theoretical expectations (Tyler 2005).

Other opportunities appear more immediately feasible: in the analysis in Vivier et al. (2004), it was shown that the ACC transport variability can be inferred from noise-free satellite magnetic data (i.e., synthetically generated magnetic data containing only the oceanic signals can be promptly inverted to regain the flow). If such a magnetic proxy of this transport were available it would be clearly adopted as an important constraint in ocean/climate models as this 
transport is both of great importance and still inadequately constrained by observations. There is indeed important debate over how this transport has varied in the glacial/interglacial past (e.g., Mazaud et al. 2010), more recent times (e.g., Whitworth and Peterson 1985; Garabato et al. 2009; Lachkar et al. 2009), and most importantly its present and expected response to changes in wind (and other) forcing associated with anthropogenic influences (e.g., Böning et al. 2008). Because the ACC is important not just as a regional phenomenon but also plays a key role in the global ocean circulation system it has received much attention. But because of its remoteness and typical under sampling in observations, an adequate descriptive and even dynamical understanding of this flow system is still forthcoming. While magnetic information about the ACC is detected and in principle available from satellite magnetometers, a sophisticated approach is required to access this information. To date, only a small fraction of the expected correlations that could be used statistically have been considered. An examination and ranking of the various observational and dynamical constraints that provide these expected correlations has not been conducted but obviously include primary dynamical assumptions arriving from both the physics and the thin-shell configuration (e.g., on large scales both the ocean flow and electric currents are confined to primarily flow "horizontally" in the relatively thin, conductive ocean shell). To give a less obvious example, consider that if nothing reliable were known about the ACC except that because of its axial alignment with regional winds, it is very efficiently wind forced [the wind work on the ACC is estimated to be about 2.1 TW (Mazlof et al. 2010)], one might draw expected relationships between the space/time distributions in wind and magnetic data sets. This approach, which has not been done so far, by-passes reliance on the modeled ocean flow and correlates magnetic data directly with the meteorological data describing ocean flow forcing (rather than the model ocean flow data).

In this study, we also assessed several exotic electromagnetic connections of the ocean with the Earth System. These primarily described the electromagnetic coupling of ocean flow and the mantle and fluid core. In all cases, the electromagnetic Lorentz forces imposed by the ocean on the other Earth elements are extremely weak. But because these forces are systematic over very long timescales, it is not immediately clear that these forces can be dismissed in all applications. Here, a closer consideration is given with quantitative estimates provided. The justification for neglecting these systematic forces cannot simply be the small amplitudes, but rather it must be shown that the amplitudes are small when compared to much stronger unresolved processes acting on the same space and time scales. As an example, it seems justified to assume that the weak axial torques on the mantle by the ocean through electromagnetic coupling may be ignored because these effects would be lost in the vagaries of oceanic mechanical torques and tidal despinning acting on similar time scales. Ignoring the forces on the fluids in the ocean and core is not as immediate. While it is certainly clear that these forces are weak when compared to the primary forces active in these fluids, it is also well known that geophysical fluids often adopt configurations where the primary forces are balanced in canceling arrangements and therefore do no work. By analogy, one could regard the wind stress on the ocean as very weak when compared to the forces associated with gravity, pressure, and rotation. For much analysis of the dynamics, one may indeed ignore the wind stress. But it is also known that the wind is in fact a primary driver of the ocean circulation, and this is revealed only once the dynamical calculations are extended beyond the dominant balance of forces. The examples above that considered Lorentz forces on the ocean and core used such an extension by positing the systematic effects of the oceanic electromagnetic forces in terms of Ekman pumping. The Ekman pumping describes the very small vertical velocity in the fluid due to the horizontal convergence of the flow generated by the forcing agent. Although the Ekman pumping is weak, it may systematically alter the dominant flows involved in the primary balance (e.g., geostrophic) through slow alteration of the geopotential surfaces. The electromagnetic Ekman pumping in the ocean and core fluids indeed seem weak even recognizing the higher level of balances that must be considered. But it is also because this higher level of balances is not yet fully understood that these exotic participants should remain within consideration.

Acknowledgements This research is supported by NASA Earth Surface and Interior Program (via geomagnetic infrastructure fund).

\section{REFERENCES}

Bloxham, J. and A. Jackson, 1991: Fluid flow near the surface of Earth's outer core. Rev. Geophys., 29, 97-120, doi: 10.1029/90RG02470. [Link]

Böning, C. W., A. Dispert, M. Visbeck, S. R. Rintoul, and F. U. Schwarzkopf, 2008: The response of the Antarctic Circumpolar Current to recent climate change. Nat. Geosci., 1, 864-869, doi: 10.1038/ngeo362. [Link]

Chave, A. D., 1983: On the theory of electromagnetic induction in the Earth by ocean currents. J. Geophys. Res., 88, 3531-3542, doi: 10.1029/JB088iB04p03531. [Link]

Cox, C. S., J. H. Filloux, and J. C. Larsen, 1971: Electromagnetic studies of ocean currents and electrical conductivity below the ocean-floor. In: Maxwell, A. E. (Ed.), The Sea, John Wiley and Sons, New York, 637-693.

Dehant, V., O. de Viron, and J. P. Barriot, 2005: Geophysical excitation of the Earth orientation parameters EOP 
and its contribution to GGOS. J. Geodyn., 40, 394-399, doi: 10.1016/j.jog.2005.06.004. [Link]

Everett, M.E. and A. Schultz, 1996: Geomagnetic induction in a heterogenous sphere: Azimuthally symmetric test computations and the response of an undulating 660km discontinuity. J. Geophys. Res., 101, 2765-2783, doi: 10.1029/95JB03541. [Link]

Everett, M. E., S. Constable, and C. Constable, 1999: Modeling three-dimensional induction effects in satellite data. Proceedings of the Second International Symposium on Three-Dimensional Electromagnetics, 63-67.

Garabato, A. C. N., L. Jullion, D. P. Stevens, K. J. Heywood, and B. A. King, 2009: Variability of Subantarctic Mode Water and Antarctic Intermediate Water in the Drake Passage during the late-twentieth and earlytwenty-first centuries. J. Climate, 22, 3661-3688, doi: 10.1175/2009JCLI2621.1. [Link]

Glazman, R. E. and Y. N. Golubev, 2005: Variability of the ocean-induced magnetic field predicted at sea surface and at satellite altitudes. J. Geophys. Res., 110, C12011, doi: 10.1029/2005JC002926. [Link]

Gross, R. S., 2009: Ocean tidal effects on Earth rotation. $J$. Geodyn., 48, 219-225, doi: 10.1016/j.jog .2009.09.016. [Link]

Gross, R. S., I. Fukumori, and D. Menemenlis, 2003: Atmospheric and oceanic excitation of the Earth's wobbles during 1980-2000. J. Geophys. Res., 108, doi: 10.1029/2002JB002143. [Link]

Gross, R. S., I. Fukumori, D. Menemenlis, and P. Gegout, 2004: Atmospheric and oceanic excitation of lengthof-day variations during 1980-2000. J. Geophys. Res., 109, B01406, doi: 10.1029/2003JB002432. [Link]

Gross, R. S., I. Fukumori, and D. Menemenlis, 2005: Atmospheric and oceanic excitation of decadal-scale Earth orientation variations. J. Geophys. Res, 110, B09405, doi: 10.1029/2004JB003565. [Link]

Gubbins, D., 2007: Geomagnetic constraints on stratification at the top of Earth's core. Earth Planets Space, 59, 661-664, doi: 10.1186/BF03352728. [Link]

Haagmans, R., 2005: Swarm: The Earth's Magnetic Field and Environment Explorers, Mission Requirements Document for Phases B, C/D, E 1 , ESTEC, Noordwijk, The Netherlands, 1-58.

Hamano, Y., T. Kasaya, H. Ichihara, and H. Tatehata, 2011: Magnetic signals from 2011 Tohoku earthquake tsunami observed at Chichijima magnetic station of JMA. Japan Geoscience Union Meeting, May 22-27, Chiba, Japan.

Helffrich, G. and S. Kaneshima, 2010: Outer-core compositional stratification from observed core wave speed profiles. Nature, 468, 807-810, doi: 10.1038/nature09636. [Link]

Holme, R., 2007: Large-scale flow in the core. In: Olson, P. (Ed.), Core Dynamics, Treatise on Geophysics, Vol. 8, Elsevier, Amsterdam, 107-130.
Holme, R. and K. A. Whaler, 2001: Steady core flow in an azimuthally drifting reference frame. Geophys. J. Int., 145, 560-569, doi: 10.1046/j.1365-246x.2001.01436.x. [Link]

Hulot, G., C. C. Finlay, C. G. Constable, N. Olsen, and M. Mandea, 2010: The magnetic field of planet earth. Space Sci. Rev., 152, 159-222, doi: 10.1007/s11214010-9644-0. [Link]

Jackson, A., 1997: Time-dependency of tangentially geostrophic core surface motions. Phys. Earth Planet.Inter., 103, 293-311, doi: 10.1016/S0031-9201(97)00039-3. [Link]

Jault, D. and J. L. Le Mouël, 1993: Circulation in the liquid core and coupling with the mantle. Adv. Space Res., 13, 221-233, doi: 10.1016/0273-1177(93)90225-Z. [Link]

Junge, A., 1988: The telluric field in northern Germany induced by tidal motion in the North Sea. Geophys. J. Int., 95, 523-533, doi: 10.1111/j.1365-246X.1988. tb06701.x. [Link]

Kelbert, A., A. Schultz, and G. Egbert, 2009: Global electromagnetic induction constraints on transition-zone water content variations. Nature, 460, 1003-1006, doi: 10.1038/nature08257. [Link]

Kherani, E. A., R. Garcia, G. Occhipinti, and P. Lognnone, 2006: Ionospheric response of the acoustic and gravity waves generated by Earthquakes and Tsunami: Linear analysis and numerical simulation. Geophys. Res. Abstr., 8.

Kuvshinov, A. V., 2008: 3-D global induction in the oceans and solid Earth: Recent progress in modeling magnetic and electric fields from sources of magnetospheric, ionospheric and oceanic origin. Surv. Geophys., 29, 139-186, doi: 10.1007/s10712-008-9045-z. [Link]

Kuvshinov, A. and A. Semenov, 2012: Global 3-D imaging of mantle electrical conductivity based on inversion of observatory $C$-responses-I. An approach and its verification. Geophys. J. Int., 189, 1335-1352, doi: 10.1111/j.1365-246X.2011.05349.x. [Link]

Lachkar, Z., J. C. Orr, J. C. Dutay, and P. Delecluse, 2009: On the role of mesoscale eddies in the ventilation of Antarctic intermediate water. Deep-Sea Res. Part IOceanogr. Res. Pap., 56, 909-925, doi: 10.1016/j. dsr.2009.01.013. [Link]

Larsen, J. and C. Cox, 1966: Lunar and solar daily variation in the magnetotelluric field beneath the ocean. J. Geophys. Res., 71, 4441-4445, doi: 10.1029/JZ071i018p04441. [Link]

Larsen, J. C., 1968: Electric and magnetic fields induced by deep sea tides. Geophys. J. Int., 16, 47-70, doi: 10.1111/j.1365-246X.1968.tb07135.x. [Link]

Larsen, J. C., 1973: An introduction to electromagnetic induction in the ocean. Phys. Earth Planet. Inter., 7, 389398, doi: 10.1016/0031-9201(73)90063-0. [Link]

Larsen, J. C. and T. B. Sanford, 1985: Florida current volume 
transports from voltage measurements. Science, 227, 302-304, doi: 10.1126/science.227.4684.302. [Link]

Lilley, F. E. M., J. H. Filloux, N. L. Bindoff, I. J. Ferguson, and P. J. Mulhearn, 1986: Barotropic flow of a warm-core ring from seafloor electric measurements. J. Geophys. Res., 91, 12979-12984, doi: 10.1029/ JC091iC11p12979. [Link]

Longuet-Higgins, M. S., M. E. Stern, and H. Stommel, 1954: The electrical field induced by ocean currents and waves, with applications to the method of towed electrodes. Papers in Physical Oceanography and Meteorology, Vol. XIII, Massachusetts Institute of Technology and Woods Hole Oceanographic Institution, doi: 10.1575/1912/1064. [Link]

Manoj,C., A. Kuvshinov, S. Maus, and H.Lühr, 2006: Ocean circulation generated magnetic signals. Earth Planets Space, 58, 429-437, doi: 10.1186/BF03351939. [Link]

Manoj, C., S. Maus, and A. Chulliat, 2011: Observation of magnetic fields generated by tsunamis. Eos, Trans., AGU, 92, 13-14, doi: 10.1029/2011EO020002. [Link]

Marcus, S. L., Y. Chao, J. O. Dickey, and P. Gegout, 1998: Detection and modeling of nontidal oceanic effects on Earth's rotation rate. Science, 281, 1656-1659, doi: 10.1126/science.281.5383.1656. [Link]

Maus, S. and A. Kuvshinov, 2004: Ocean tidal signals in observatory and satellite magnetic measurements. Geophys. Res. Lett., 31, L15313, doi: 10.1029/2004GL020090. [Link]

Mazaud, A., E. Michel, F. Dewilde, and J. L. Turon, 2010: Variations of the Antarctic Circumpolar Current intensity during the past $500 \mathrm{ka}$. Geochem. Geophys. Geosyst., 11, Q08007, doi: 10.1029/2010GC003033. [Link]

Mazlof, M. R., P. Heimbach, and C. Wunsch, 2010: An eddy-permitting southern ocean state estimate. J. Phys. Oceanogr., 40, 880-899, doi: 10.1175/2009JPO4236.1. [Link]

McKnight, J. D., 1995: Lunar daily geomagnetic variations in New Zealand. Geophys. J. Int., 122, 889-898, doi: 10.1111/j.1365-246X.1995.tb06844.x. [Link]

Occhipinti, G., E. A. Kherani, P. Lognonné, 2008: Geomagnetic dependence of ionospheric disturbances induced by tsunamigenic internal gravity waves. Geophys. J. Int., 173, 753-765, doi: 10.1111/j.1365246X.2008.03760.x. [Link]

Occhipinti, G., L. Rolland, P. Lognonné, and S. Watada, 2013: From Sumatra 2004 to Tohoku-Oki 2011: The systematic GPS detection of the ionospheric signature induced by tsunamigenic earthquakes. J. Geophys. Res., 118, 3626-3636, doi: 10.1002/jgra.50322. [Link]

Olsen, N. and M. Mandea, 2008: Rapidly changing flows in the Earth's core. Nat. Geosci., 1, 390-394, doi: 10.1038/ngeo203. [Link]

Olsen, N., K. H. Glassmeier, and X. Jia, 2010: Separation of the magnetic field into external and internal parts.
Space Sci. Rev., 152, 135-157, doi: 10.1007/s11214009-9563-0. [Link]

Peltier, W. R. and R. Drummond, 2010: Deepest mantle viscosity: Constraints from earth rotation anomalies. Geophys. Res. Lett., 37, L12304, doi: 10.1029/2010GL043219. [Link]

Podney, W., 1975: Electromagnetic fields generated by ocean waves. J. Geophys. Res., 80, 2977-2990, doi: 10.1029/JC080i021p02977. [Link]

Ryskin, G., 2009: Secular variation of the Earth's magnetic field: Induced by the ocean flow? New J. Phys., 11, doi: 10.1088/1367-2630/11/6/063015. [Link]

Salstein, D. A., D. M. Kann, A. J. Miller, and R. D. Rosen, 1993: The Sub-bureau for Atmospheric Angular Momentum of the International Earth Rotation Service: A meteorological data center with geodetic applications. Bull. Amer. Meteorol.Soc., 74, 67-80, doi: 10.1175/15 20-0477(1993)074<0067:TSBFAA>2.0.CO;2. [Link]

Sanford, T. B., 1971: Motionally induced electric and magnetic fields in the sea. J. Geophys. Res., 76, 3476-3492, doi: 10.1029/JC076i015p03476. [Link]

Semenov, A. and A. Kuvshinov, 2012: Global 3-D imaging of mantle conductivity based on inversion of observatory $C$-responses-II. Data analysis and results. Geophys. J. Int., 191, 965-992, doi: 10.1111/j.1365246X.2012.05665.x. [Link]

Stephenson, D. and K. Bryan, 1992: Large-scale electric and magnetic fields generated by the oceans. J. Geophys.Res., 97, 15467-15480, doi: 10.1029/92JC01400. [Link]

Stephenson, F. R. and L. V. Morrison, 1995: Long-term fluctuations in the Earth's rotation: $700 \mathrm{BC}$ to $\mathrm{AD}$ 1990. Phil. Trans. Roy. Soc. Lond., 351, 165-202, doi: 10.1098/rsta.1995.0028. [Link]

Toh, H., K. Satake, Y. Hamano, Y. Fujii, and T. Goto, 2011: Tsunami signals from the 2006 and 2007 Kuril earthquakes detected at a seafloor geomagnetic observatory. J. Geophys. Res., 116, B02104, doi: 10.1029/2010JB007873. [Link]

Tyler, R. H., 1995: Electromagnetic fields generated by ocean currents and the potential for using geomagnetic data in ocean and climate studies. Ph.D. Thesis, McGill University, Montreal, Canada.

Tyler, R. H., 2005: A simple formula for estimating the magnetic fields generated by tsunami flow. Geophys. Res. Lett., 32, L09608, doi: 10.1029/2005GL022429. [Link]

Tyler, R. H., 2006: Weak influences of the Earth's magnetic field on ocean circulation. Geophys. Res. Lett., 33, L14615, doi: 10.1029/2006GL026372. [Link]

Tyler, R. H. and R. Käse, 2000a: A numerical study of the string function using a primitive equation ocean model. Geophys. Astro. Fluid, 92, 65-83, doi: 10.1080/03091920008203711. [Link] 
Tyler, R. H. and R. Käse, 2000b: A ‘string function' for describing the propagation of large-scale potential energy anomalies in a rotating fluid. Geophys. Astro. Fluid, 92, 31-64, doi: 10.1080/03091920008203710. [Link]

Tyler, R. H. and R. Käse, 2001: A string function for describing the propagation of baroclinic anomalies in the ocean. J. Phys. Oceanogr., 31, 765-776, doi: 10.1175/1 520-0485(2001)031<0765:ASFFDT>2.0.CO;2. [Link]

Tyler, R. H. and L. A. Mysak, 1995: Motionally-induced electromagnetic fields generated by idealized ocean currents. Geophys. Astro. Fluid, 80, 167-204, doi: 10.1080/03091929508228954. [Link]

Tyler, R. H. and T. B. Sanford, 1998: Electromagnetic forces on the earth's core due to the poleward transport of heat in the oceans. Geophys. Astro. Fluid, 88, 115-129, doi: 10.1080/03091929808245470. [Link]

Tyler, R. H., L. A. Mysak, and J. M. Oberhuber, 1997a: Electromagnetic fields generated by a three dimensional global ocean circulation. J. Geophys. Res., 102, 5531-5551, doi: 10.1029/96JC03545. [Link]

Tyler, R. H., T. B. Sanford, and J. M. Oberhuber, 1997b: Geophysical challenges in using large-scale oceangenerated EM fields to determine the ocean flow. $J$. Geomagn. Geoelectr., 49, 1351-1372, doi: 10.5636/ jgg.49.1351. [Link]

Tyler, R. H., J. M. Oberhuber, and T. B. Sanford, 1999: The potential for using ocean generated electromagnetic fields to remotely sense ocean variability. Phys. Chem. Earth, 24, 429-432, doi: 10.1016/S14641895(99)00054-X. [Link]

Tyler, R. H., S. Maus, and H. Lühr, 2003: Satellite observations of magnetic fields due to ocean tidal flow. Science, 299, 239-241, doi: 10.1126/science.1078074. [Link]

Tyler, R. H., F. Vivier, and S. Li, 2004: Three-dimen- sional modelling of ocean electrodynamics using gauged potentials. Geophys. J. Int., 158, 874-887, doi: 10.1111/j.1365-246X.2004.02318.x. [Link]

Utada, H., H. Shimizu, T. Ogawa, T. Maeda, T. Furumura, T. Yamamoto, N. Yamazaki, Y. Yoshitake, and S. Nagamachi, 2011: Geomagnetic field changes in response to the 2011 off the Pacific Coast of Tohoku Earthquake and Tsunami. Earth Planet. Sci. Lett., 311, 11-27, doi: 10.1016/j.epsl.2011.09.036. [Link]

Vennerstrom, S., E. Friis-Christensen, H. Lühr, T. Moretto, N. Olsen, C. Manoj, P. Ritter, L. Rastätter, A. Kuvshinov, and S. Maus, 2005: Swarm: The impact of combined magnetic and electric field analysis and of ocean circulation effects on Swarm mission performance. Doc. No. (Issue): SWSC/DSRI/MIS/TN/0001(2), Final Report, Danish Space Research Institute, 1-139.

Vivier, F., E. Maier-Reimer, and R. H. Tyler, 2004: Simulations of magnetic fields generated by the Antarctic Circumpolar Current at satellite altitude: Can geomagnetic measurements be used to monitor the flow? Geophys. Res. Lett., 31, L10306, doi: 10.1029/2004GL019804. [Link]

Weaver, J. T., 1965: Magnetic variations associated with ocean waves and swell. J. Geophys. Res., 70, 19211929, doi: 10.1029/JZ070i008p01921. [Link]

Whaler, K. A. and R. Holme, 2007: Consistency between the flow at the top of the core and the frozen-flux approximation. Earth Planets Space, 59, 1219-1229, doi: 10.1186/BF03352070. [Link]

Whitworth, T. and R. G. Peterson, 1985: Volume transport of the Antarctic Circumpolar Current from bottom pressure measurements. J. Phys. Oceanogr., 15, 810816, doi: 10.1175/1520-0485(1985)015<0810:VTOTAC>2.0.CO;2. [Link] 